\title{
Modeling of 3D Scene Based on Series of Photographs Taken with Different Depth-of-Field
}

\author{
Marcin Denkowski $^{1}$, Michał Chlebiej ${ }^{2}$, and Paweł Mikołajczak ${ }^{1}$ \\ ${ }^{1}$ Faculty of Computer Science, Maria Curie-Skłodowska University, \\ pl. Marii Curie-Skłodowskiej 5, 20-031 Lublin, Poland \\ denmar@goblin.umcs.lublin.pl \\ ${ }^{2}$ Faculty of Mathematics and Computer Science, N. Copernicus University, \\ Chopina 12/18, 87-100 Toruń, Poland
}

\begin{abstract}
This paper presents a method for fusing multifocus images into enhanced depth-of-field composite image and creating a 3D model of a photographed scene. A set of images of the same scene is taken from a typical digital camera with macro lenses with different depth-of-field. The method employs convolution and morphological filters to designate sharp regions in this set of images and combine them together into an image where all regions are properly focused. The presented method consists of several phases including: image registration, height map creation, image reconstruction and final $3 \mathrm{D}$ scene reconstruction. In result a $3 \mathrm{D}$ model of the photographed object is created.
\end{abstract}

\section{Introduction}

Macro photography is a type of close-up photography with magnification ratios from about 1:1 to about 10:1. The most crucial parameter of macro photography is the depth of field (DOF) [1. Because it is very difficult to obtain high values of DOF for extreme close-ups it is essential to focus on the most important part of the subject. Any other elements that are even a millimeter farther or closer may appear blurred in the acquired photo. The depth of field can be defined as the distance in front of and behind the subject appearing in focus. Only a very short range of the photographed subject will appear in exact focus. The most important factor that determine whether the subject appears in focus is how a single point is mapped onto the sensor area. If a given point is exactly at the focus distance it will be imaged as one point on the sensor, in the other case it will produce a disk whose border is known as a "circle of confusion". These circles can be used to define the measure of focus and blurriness as they increase in diameter the further away they are from the focus point. For a specific film format, the depth of field is described as a function parametrized by: the focal length of the lens, the diameter of the lens opening (the aperture), and the distance between the subject and the camera. Let D be the distance at which the camera is focused, $\mathrm{F}$ the focal length (in millimeters) calculated for

M. Bubak et al. (Eds.): ICCS 2008, Part II, LNCS 5102, pp. 25-34, 2008.

(C) Springer-Verlag Berlin Heidelberg 2008 
an aperture number $\mathrm{f}$ and $\mathrm{k}$ - the "circle of confusion" for a given film format (in millimeters), then depth of field (DOF) [1] can be defined as:

$$
D O F_{1,2}=\frac{D}{1 \pm \frac{1000 \times D \times k \times f}{F 2}}
$$

where $D O F_{1}$ is distance from the camera to the far depth of field limit, and $D O F_{2}$ is the distance from the camera to the near depth of field limit. The aperture controls the effective diameter of the lens opening. Reduction of the aperture size increases the depth of field, however, it also reduces the amount of light transmitted. Lenses with a short focal length have a greater depth-offield than long lenses. Greater camera-to-subject distance results in a greater depth-of-field.

We used this optical phenomenon to achieve two aims. The first one was to obtain the deepest possible depth-of-field using standard digital camera images and image processing algorithms. And the second goal was to create threedimensional model of photographed scene.

As an input we have created a series of macro photograph images of the same subject with different focus lengths. In the first step of our method we have to register them together to create a properly aligned stack of images. The next step is to fuse them into a one composit image. For that purpose we propose enhanced multiscale convolution and morphology method, which we have introduced in 2. Methods for image fusion using multiscale morphology have been broadly discussed in 345. As an effect of fusing algorithm we obtain a height map and the reconstructed focused image with a very deep depth-offield. The height map is a label map which determines the height of each part of the scene. From this map, we can construct a 3D model of the scene. In this work we limit our method to macro photography only and we assume that images were taken perpendicularly or almost perpendicularly to the scene. However, to clearly present advantages and problems of our method, we also show some cases with sets of images acquired in different way.

\section{Registration}

In the first step a set of photographs of the desire object is acquired. Unfortunately, during extreme close-up sessions small movements of the camera are possible even when using tripods for stabilization. To make the reconstruction method more robust we can make use of an image registration procedure. The main idea behind image registration is to find perfect geometric alignment between a set of overlapping images. The quality of match measure represents the matching function parametrized by the geometric transformation. In our method we use the rigid (translations and rotation) or the affine transformation model (rigid + scaling and shears). In most cases it is sufficient to use the simplified rigid transformation (translations only). But when images are acquired without stabilization devices the use of complete affine transformation is a necessity. In 
our approach we use the normalized mutual information [7] as the matching function:

$$
N M I(F I, R I)=\frac{h(F I)+h(R I)}{h(F I, R I)}
$$

where $R I$ represents the reference image and $F I$ represents the floating image.

$$
\begin{gathered}
h(F I)=-\sum p_{F I}(x) \log \left(p_{F I}(x)\right) \\
h(R I)=-\sum p_{R I}(x) \log \left(p_{R I}(x)\right) \\
h(F I, R I)=-\sum \sum p_{F I, R I}(x, y) \log \left(p_{F I, R I}(x, y)\right)
\end{gathered}
$$

where $h(F I), h(R I)$ and $h(F I, R I)$ are the single and joint entropies [2], $p_{F I}$ and $p_{R I}$ are the probabilities of each intensity in the intersection volume of both data sets and $p_{F I, R I}$ is a probability distribution of a joint histogram. For the minimization of the selected similarity measure we use Powell's algorithm 8]. As a result of the registration procedures we obtain a set of geometrically matched images that can be used in the next stages of our wide depth of field reconstruction algorithm.

\section{Image Fusion}

\subsection{Overview}

Image fusion is a process of combining a set of images of the same scene into one composite image. The main objective of this technique is to obtain an image that is more suitable for visual perception. This composite image has reduced uncertainty and minimal redundancy while the essential information is maximized. In other words, image fusion integrates redundant and complementary information from multiple images into a composite image but also decreases dimensionality. There are many methods discovered and discussed in literature that focus on image fusion. They vary with the aim of application used, but they can be mainly categorized due to algorithms used into pyramid techniques [1011], morphological methods [345], discrete wavelet transform [12 1314] and neural network fusion [15.

The different classification of image fusion involves pixel, feature and symbolic levels [16. Pixel-level algorithms are low level methods and work either in the spatial or in transform domain. This kind of algorithms work as a local operation despite of transform used and can generate undesirable artifacts. These methods can be enhanced by using multiresolution analysis [10] or by complex wavelet transform [14. Feature-based methods use segmentation algorithms to divide images into relevant patterns and then combine them to create output image by using various properties [17. High-level methods combine image descriptions, typically, in the form of relational graphs [18]. 


\subsection{Methodology}

In our work we use multiscale convolution and morphology methods combined with pyramid segmentation algorithm to distinguish homogeneous regions. Our fusion method is also capable to work with color images. Color image fusion has been discussed in [19. At this stage we assume that images on the image stack are aligned to each other. At this point the main objective is to create focused image and the height map. The whole algorithm, shown in Fig. 1, can be divided into 5 stages:

1. Creation of n-level multiresolution pyramid for every input image. In this case we use median filter to downscale images.

2. Segmentation of every image on the stack by using pyramid segmentation. For this process we convert images into HSL color model 9 to separate luminance (contrast) information contained in luminance channel from color description in hue and saturation channels. Example results of the segmentation process are shown in Fig. 2] as segmentation maps.

3. Calculation of local standard deviation $S D$ at local region $R$ for every pixel $f(x, y)$ at each pyramid level $L$ for every image on the stack $(z)$ :

$$
S D_{R}^{(L)}(x, y, z)=\sqrt{\frac{1}{N_{R}} \sum_{(x, y) \in R, z}\left(f(x, y)-\overline{f_{R}}\right) 2}
$$

Color RGB components are coverted to its graylevel intensity according to $G_{f}=0.299 R+0.587 G+0.114 B$.

4. Reconstruction rules.

Step-1. For the lowest level of pyramid, pixels with maximum $S D_{\max }^{(0)}(x, y, z)$ are marked as focus and labeled in the height map $H M(x, y)$ with $z$ value. If $\operatorname{abs}\left(S D_{\max }^{(0)}(x, y)-S D_{\min }^{(0)}(x, y)\right)<T_{s}$, where $T_{s}$ is a threshold value, pixel is marked as unresolved because it usually belongs to smooth region. These pixels are taken care of at subsequent steps.

Step-2. Every pixel is checked with the segmentation map. If it isn't nearby any edge and its $S D_{R}(x, y, z)$ value drastically differ from $S D_{R}(x, y, z)$ average pixel value for its region $R$ it is marked with $S D_{R}(x, y, z)$ value of the median pixel. It prevents from marking false or noise pixels.

Step-3. For every i-th pyramid level, starting from $\mathrm{i}=1$, if $S D_{R}^{(i)}(x, y, z)$ of actual pixel is not equal to $S D_{R}^{(i-1)}(x, y, z)$ from previous pyramid level, then:

(a) if the pixel is nearby some edge marked on the segmentation map, pixel with $\max \left(S D_{R}^{(i)}(x, y, z), S D_{R}^{(i-1)}(x, y, z)\right)$ value is taken and labeled in the height map $H M(x, y)$ with $(i)$ or $(i-1)$ value,

(b) else, the height map $H M(x, y)$ is labeled as:

$$
H M(x, y)=H M^{(i-1)}(x, y)+\frac{H M^{(i)}(x, y)-H M^{(i-1)}(x, y)}{2}
$$




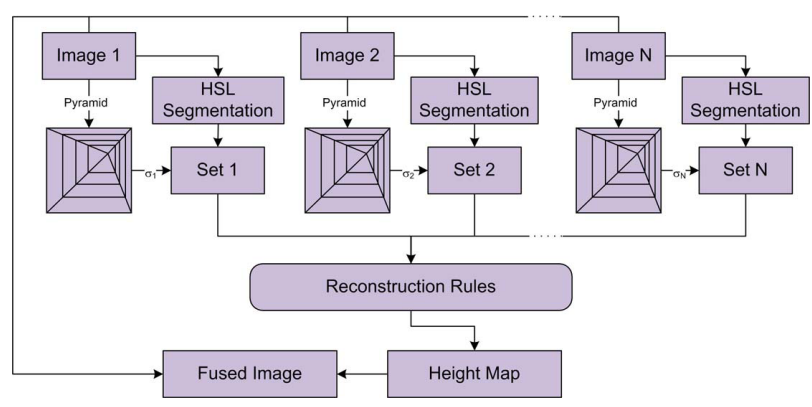

Fig. 1. Image Fusion scheme using pyramid decomposition and HSL Segmentation

Step-4. Labeling remaining pixels. If unresolved pixel belongs to region with many other unresolved pixels it is marked as a background, else the median value from region is taken.

5. Creation of fusing image. The value of fused image pixel $f(x, y)$ is equal to the pixel $f^{(z)}(x, y)$ from $z-t h$ input image on the stack, where $z$ is a value taken from created height map $H M(x, y)$.

The main difficulty is to obtain the height map without spikes or noise, generally smooth but with sharp edges. It is not essential from the point of view of the image fusion, but it may be crucial in three-dimensional reconstruction of the scene. Most of such peaks are generated in smooth regions, where noise in defocused region on one image from the stack often gives greater values of $S D$ than in the corresponding region on sharp image. This leads to undesired deformations of reconstructed spatial surface. For that reason, it is necessary to determine a background plane. For now, we assumed that the background plane overlaps with the last image on the stack, but the plane equation may be also given by hand.

Fusion process often creates halo effects near the edges of objects. This phenomenon can be observed in Fig. 3. To resolve this problem we use segmentation maps to determine edges. After that we are able to mark pixels near edges properly as shown in Step-2 and Step-3 of Reconstruction rules.

\section{3D Scene Creation}

Spatial scene is generated on the basis of information contained in the height map, where each pixel value represents $z$ coordinate of appropriate mesh vertex. In $3 \mathrm{D}$ reconstruction process we have considered two methods e.i. marching cubes algorithm (MS) 2021] and simply by changing $z$ coordinate in a 3D regular mesh. Both methods have advantages as well as disadvantages. Marching-cube gives more control over reconstruction process but is also more complicated and sometimes produces too sharp blocky edges, while second method is very simple and fast but always produces regular mesh. Generated mesh is decimated and smoothed. Created surface is textured with a plane mapping by the fused image. 


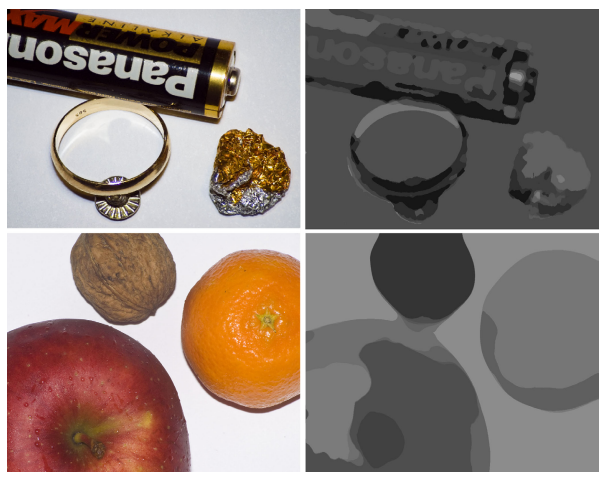

Fig. 2. Segmentation maps created by using pyramid segmentation (right column) for multifocus images (left column)

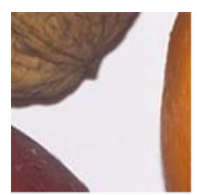

(a)

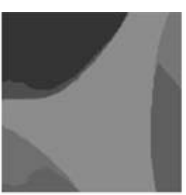

(b)

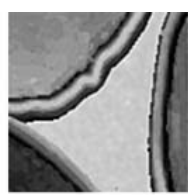

(c)

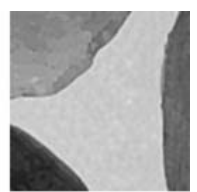

(d)

Fig. 3. Example of halo effect. Part of the original image (a), the segmentation map (b), the height map created without using the segmentation map - visible halo effect (c) and edges in the height map with help of the segmentation map (d).

\section{Experimental Results}

The proposed method has been implemented on Linux platform in $\mathrm{C}++$ language using SemiVis framework 22 and Kitware VTK library for visualisation purposes. For testing procedure we have prepared eight image stacks from macrophotography. Each stack contains six to twelve images taken with different depth-of-field, and one control image taken with the largest possible depth-of-field that we were able to receive from our testing digital camera with macro lens.

In all cases the procedure is performed in the following order. At first, the registration process aligns multifocus images to each other to minimize misregistration. Then all images are segmented and the pyramid is created up to three levels. Finally, the reconstruction process combine image stack into height map and fused image.

Reconstruction time strongly depends on the size of the images used in the fusion and the number of images on the stack. The most computationally expensive is the registration procedure, which consumes above fifty percent of the overal reconstruction time. The fusion process takes about 35\%, and generation of three dimensional mesh takes remaining $15 \%$. For a typical set of images, containing ten images with resolution $512 \times 512$ the whole procedure lasts about 60 seconds. 


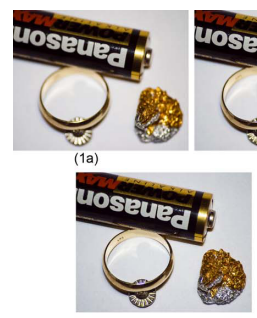

(1e)
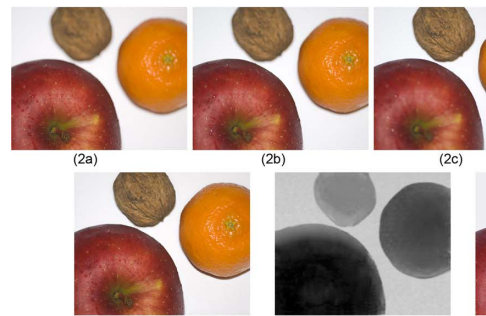

(2e)

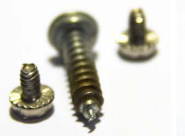

(3a)

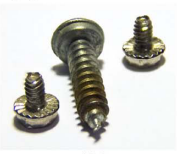

(3e)

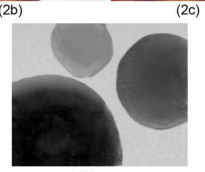

(2f)

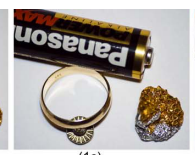

(1c)

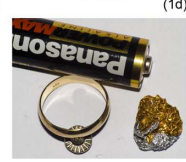

(19)
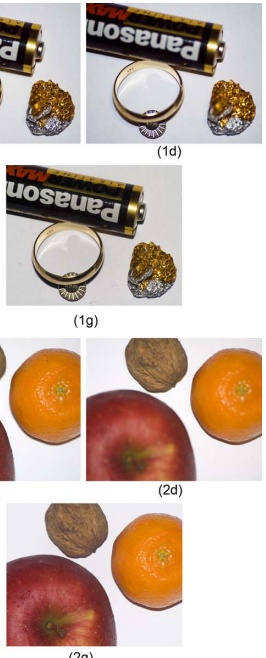

(2g)

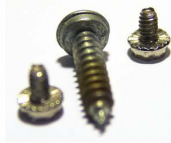

(3c)

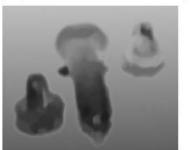

(3f)

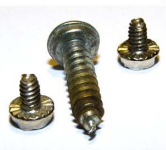

$(3 g)$

Fig. 4. Sets of multifocus images $(1,2,3 \mathrm{abc})$, reconstructed focus image $(1,2,3 \mathrm{e})$, created height map (1,2,3f), control image taken with the largest possible depth-of-field $(1,2,3 \mathrm{~g})$

Examples of multifocus images with height map and reconstructed fused images are shown in Fig. 4. Each fused image is compared to its control image. Mutual Information (MI) and Mean Square Difference (MSD) are useful tools in such comparison. Table 1 contains calculated similarity values for every fused image and corresponding reference image.

Table 1 also contains widely used metric $Q^{A B / F}$ that measures quality of image fusion. This measure was proposed by Xydeas and Petrović in [23. In this case, a per-pixel measure of information preservation is obtained between each input and the fused image which is aggregated into a single score $Q^{A B / F}$ using a simple local importance assignment. This metric is based on the assumption that fusion algorithm that transfers input gradient information into result image more accuretely performs better. $Q^{A B / F}$ is in range $[0,1]$ where 0 means complete loss of information and 1 means perfect fusion.

From the height map and fused image we can generate 3D model of the scene. Additionally, the height map is filtered with strong median and gaussian filter 
Table 1. Similarity measures between reconstructed image and reference image with large depth-of-field - MI and MSD and the quality measure $Q^{A B / F}$

\begin{tabular}{l||cc|c}
\hline \multirow{2}{*}{ Stack } & \multicolumn{2}{|c|}{ Similarity measures } & \multirow{2}{*}{$Q^{A B / F}$} \\
\cline { 2 - 3 } & MI & MSD & \\
\hline S-1 & 0.82 & 28.48 & 0.84 \\
S-2 & 0.67 & 32.11 & 0.73 \\
S-3 & 0.72 & 38.43 & 0.79 \\
S-4 & 0.88 & 26.03 & 0.85 \\
S-5 & 0.82 & 27.74 & 0.80 \\
S-6 & 0.64 & 35.81 & 0.72 \\
S-7 & 0.69 & 34.30 & 0.72 \\
S-8 & 0.71 & 41.65 & 0.69 \\
\hline
\end{tabular}
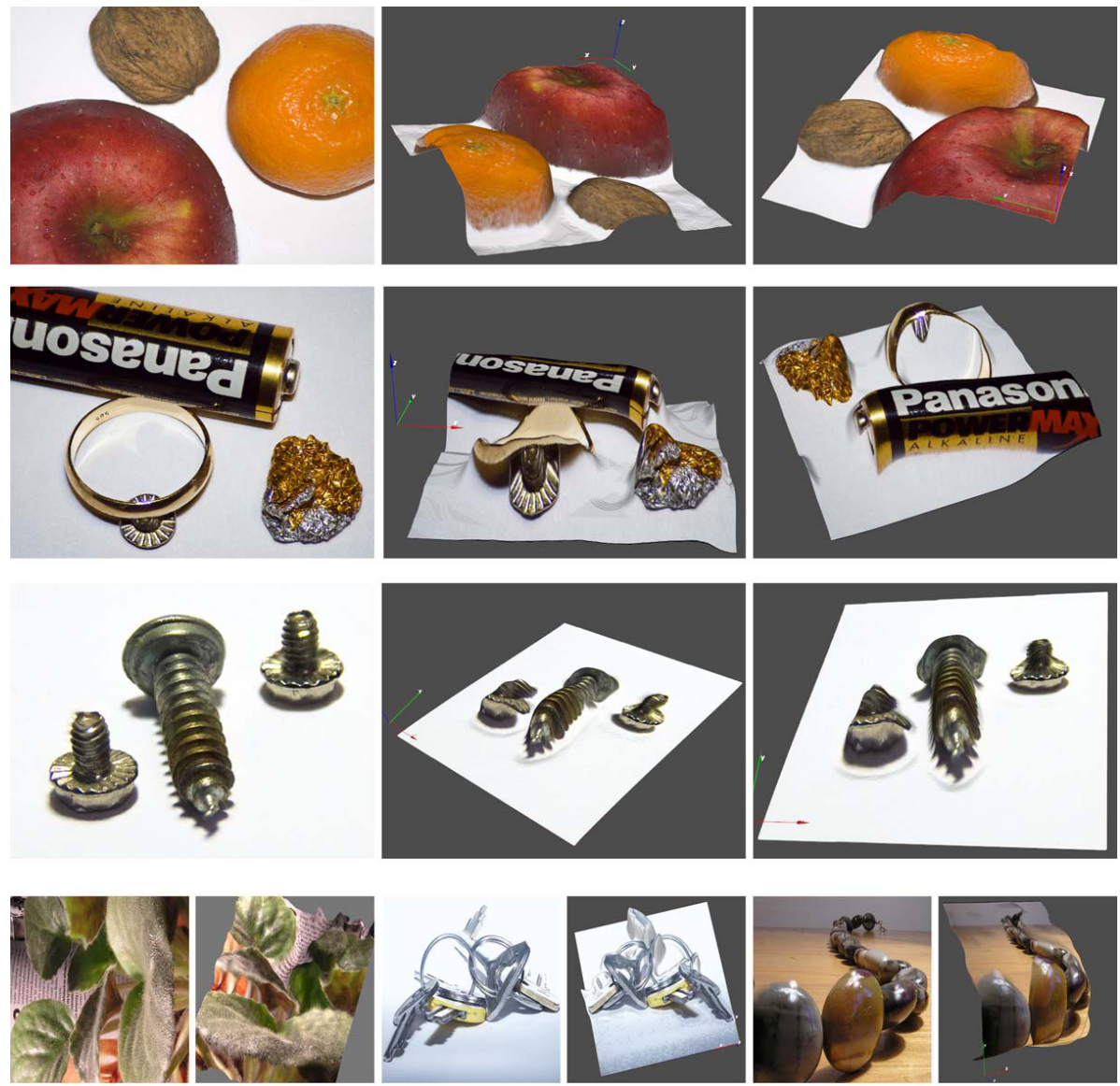

Fig. 5. Result fused images and 3D models 

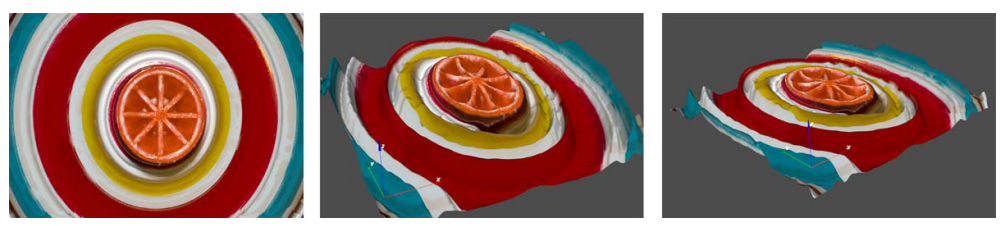

Fig. 6. Typical image that creates failed 3D model. This photograph presents a common child's spinning top. Reconstruction algorithms failed because of many smooth and uniform regions and a lack of background plane.

to smooth regions and after that the mesh is created. Fig. 5 shows qualitative results of our method for eight tested image sets. The biggest problem in this $3 \mathrm{~d}$ reconstruction is to obtain a surface which is smooth enough in uniform regions and simultaneously has sharp edges on the objects boundaries. The best results are received when the photographs are taken perpendicularly to the background, objects are within the scene, and they are rough without smooth regions.

Fig. 6] shows an example of a typical failure. Our method often fails when there are large smooth regions which don't belong to the background plane. The main difficulty in such cases is to distinguish between background and an object without any external spatial knowledge of the scene.

\section{Conclusions}

This paper presented an attempt to the problem of generating of $3 \mathrm{~d}$ model from a set of multifocus images. We proposed the whole pipeline from raw photographs to the final spatial model. Input multifocus images were registered together and next, using typical image filters and gradient methods the height map was created by detecting focused regions in each of them. Based on the height map the image with a greater depth-of-field was composed. Finally, further algorithms reconstructed the $3 \mathrm{~d}$ model of the photographed scene.

The presented results of generation of $3 \mathrm{D}$ models are very promising, but as for now, there are still many problems that need to be solved. Future work could include improvements in segmentation and edge detection to help in automatic detection of the background plane. Second, there should be more complex methods used to identify smooth regions of objects. We think that in both cases pattern recognition algorithms should improve effectiveness of our method. Also Feature-based fusion methods such as [17] could generate more accurate height maps.

\section{References}

1. Constant, A.: Close-up Photography. Butterworth-Heinemann (2000)

2. Denkowski, M., Chlebiej, M., Mikołajczak, P.: Depth of field reconstruction method using partially focused image sets. Polish Journal of Environmental Studies 16(4A), 62-65 (2007) 
3. Ishita, D., Bhabatosh, C., Buddhajyoti, C.: Enhancing effective depth-of-field by image fusion using mathematical morphology. Image and Vision Computing 24, 1278-1287 (2006)

4. Mukopadhyay, S., Chanda, B.: Fusion of 2d gray scale images using multiscale morphology. Pattern Recognition 34, 1939-1949 (2001)

5. Matsopoulos, G.K., Marshall, S., Brunt, J.N.M.: Multiresolution morphological fusion of $\mathrm{mr}$ and ct images of the human brain. IEEE Proceedings Vision, Image and Signal Processing 141(3), 137-142 (1994)

6. Eltoukhy, H., Kavusi, S.: A computationally efficient algorithm for multi-focus image reconstruction. In: Proceedings of SPIE Electronic Imaging (June 2003)

7. Studholme, C., et al.: An overlap invariant entropy measure of 3D medical image alignment. Pattern Recognition 32(1), 71-86 (1999)

8. Press, W.H., Flannery, B.P., Teukolsky, S.A., Vetterling, W.T.: Numerical Recipes in C, 2nd edn. Cambridge University Press, Cambridge (1992)

9. Gonzalez, R.C., Woods, R.E.: Digital image processing. Addison-Wesley Publishing Company, Inc, Reading (1992)

10. Burt, P.J.: The pyramid as a structure for efficient computation. In: Multiresolution Image Processing and Analysis, pp. 6-35. Springer, Berlin (1984)

11. Toet, A.: Image fusion by rati of low-pass pyramid. Pattern Recognition Letters $9(4), 245-253(1989)$

12. Li, H., Manjunath, H., Mitra, S.: Multisensor image fusion using the wavelet transform. Graphical Models and Image Processing 57(3), 235-245 (1995)

13. Chibani, Y., Houacine, A.: Redundant versus orthogonal wavelet decomposition for multisensor image fusion. Pattern Recognition 36, 879-887 (2003)

14. Lewis, L.J., O'Callaghan, R., Nikolov, S.G., Bull, D.R., Canagarajah, N.: Pixeland region-based image fusion with complex wavelets. Information Fusion 8, 119 130 (2007)

15. Ajjimarangsee, P., Huntsberger, T.L.: Neural network model for fusion of visible and infrared sensor outputs, Sensor Fusion, Spatial Reasoning and Scene Interpretation. In: The International Society for Optical Engineering, SPIE, Bellingham, USA, vol. 1003, pp. 152-160 (1988)

16. Goshtasby, A.A.: Guest editorial: Image fusion: Advances in the state of the art. Information Fusion 8, 114-118 (2007)

17. Piella, G.: A general framework for multiresolution image fusion: from pixels to regions. Information Fusion 4, 259-280 (2003)

18. Wiliams, M.L., Wilson, R.C., Hancock, E.R.: Deterministic search for relational graph matching. Pattern Recognition 32, 1255-1516 (1999)

19. Bogoni, L., Hansen, M.: Pattern-selective color image fusion. Pattern Recognition 34, 1515-1526 (2001)

20. Lorensen, W.E., Cline, H.E.: Marching cubes: A high resolution 3D surface construction algorithm. Computer Graphics 21(4), 163-169 (1987)

21. Durst, M.J.: Additional reference to Marching Cubes. Computer Graphics 22(2), $72-73$ (1988)

22. Denkowski, M., Chlebiej, M., Mikołajczak, P.: Development of the cross-platform framework for the medical image processing. Annales UMCS, Sectio AI Informatica III, 159-167 (2005)

23. Xydeas, C., Petrović, V.: Objective image fusion performance measure. Electronics Letters 36(4), 308-309 (2000) 